\title{
DIAGNÓSTICOS DE ENFERMAGEM IDENTIFICADOS EM IDOSOS HOSPITALIZADOS: ASSOCIAÇÃO COM AS SÍNDROMES GERIÁTRICAS a
}

\author{
Nursing diagnoses identified in hospitalized elderly: association with geriatrics' \\ syndromes

\section{Diagnósticos de enfermería identificados en ancianos hospitalizados: asociación con las síndromes geriátricas}

Renata Miranda de Sousa ${ }^{1}$

Rosimere Ferreira Santana²

Fátima Helena do Espírito Santo 3
Luise de Almeida Ferreira Alves ${ }^{5}$

\section{RESUMO}

Identificar e analisar os diagnósticos de enfermagem associados à presença das síndromes geriátricas em idosos hospitalizados. MÉTODOS: Trata-se de um estudo de abordagem quantitativo, utilizando-se das técnicas de pesquisa, entrevista semiestruturada e formulários específicos da gerontologia. Os sujeitos foram 66 pessoas com mais de 65 anos, internadas nas unidades clínicas e cirúrgicas do Hospital Universitário Antonio Pedro, Niterói-RJ, Brasil. RESULTADOS: Identificamos 394 diagnósticos de enfermagem correlacionados às cinco principais síndromes geriátricas, Isolamento Social $129(32,8 \%)$, latrogenia $113(28,6 \%)$, Instabilidade Postural 81 (20,6\%), Insuficiência cerebral 44 (11,1\%) e Incontinência urinária 27 (6,9\%). DISCUSSÃ0: Podemos inferir que em média ocorrem duas síndromes por idoso, aumentando o risco de fragilização e, consequentemente, a chance de complicações, aumento do custo e reinternações. CONCLUSÃO: 0 cuidado de enfermagem direcionado à identificação dos riscos potenciais pode contribuir para a promoção da independência e autonomia, para a redução de complicações, para o tempo de hospitalização e futuras reinternações.

Palavras-chave: Diagnóstico de Enfermagem. Saúde do Idoso. Enfermagem Geriátrica.

\section{Abstract}

OBJECTIVE: Identify and analyze the nursing diagnoses relates to the presence of geriatric syndromes in hospitalized elderly. METHODS: This is a study of a quantitative approach, using researching techniques, semi-structured interviews and specific forms from gerontology. The subjects were 66 individuals over 65 years old, admitted to the clinical and surgical units of the University Hospital Antonio Pedro, Niterói-RJ, Brazil. RESULTS: We identified 394 nursing diagnoses related to five major geriatric syndromes, Social Isolation 129 (32,8\%), latrogenic 113 (28,6\%), Postural Instability 81 (20,6\%) Cerebral Insufficiency $44(11,1 \%)$, and Urinary Incontinence $27(6,9 \%)$. DISCUSSION: We can infer that on average two syndromes occur in elderly, increasing the risk of weakness, and consequently the likelihood of complications, increased cost, and re-admissions. CONCLUSION: Therefore nursing care for hospitalized elderly should be directed to facilitate the identification of potential risks, in order to promote independence and the maintenance of autonomy, to reduce complications, hospitalization time and future re-admissions.

Keywords: Nursing Diagnosis. Health of the Elderly. Geriatric Nursing.

\section{Resumen}

OBJETIVO: Identificar y analizar los diagnósticos de enfermería relacionados con la presencia de síndromes geriátricos en ancianos hospitalizados. MÉTODOS: Este es un estudio de enfoque cuantitativo, utilizando las técnicas de investigación, las formas semi-estructuradas y específicas de Gerontología, los sujetos fueron 64 personas con más de 65 años ingresados en las unidades clínicas e quirúrgicas del Hospital Universitario Antonio Pedro, Niterói-RJ, Brasil. RESULTADOS: Se identificaron 394 diagnósticos de enfermería relacionados con cinco grandes síndromes geriátricos, Aislamiento Social 129 (32,8\%), latrogenia 113 (28,6\%), Inestabilidad Postural $81(20,6 \%)$ Insuficiencia Cerebral $44(11,1 \%)$ y Incontinencia Urinaria $27(6,9 \%)$. DISCUSIÓN: Podemos inferir que en promedio dos síndromes se producen en personas de edad avanzada, aumentando el riesgo de debilidad, y por lo tanto la probabilidad de complicaciones, mayor costo, y las readmisiones. CONCLUSIÓN: Por lo tanto la atención de enfermería para ancianos hospitalizados dirigidas a facilitar la identificación de riesgos potenciales, con el fin de propiciar la independencia y el mantenimiento de la autonomía, para reducir las complicaciones, el tiempo de hospitalización y futuros reingresos.

Palabras clave: Diagnóstico de Enfermería. Salud del Anciano . Enfermería Geriátrica .

'Enfermeira. Residente em Enfermagem Médico-Cirúrgico pela Universidade Federal do Estado do Rio de Janeiro (UNIRIO). Bolsista FAPERJ. Membro do Núcleo de Estudos e Pesquisa em Enfermagem Gerontológica da Escola de Enfermagem Aurora de Afonso Costa da Universidade Federal Fluminense (NEPEG/EEAAC/UFF). Rio de Janeiro, R. Brasil. E-mail: natinha.sousa@yahoo.com.br.'2Enfermeira. Doutora em Enfermagem. Especialista em Psicogeriatria. Professora Adjunta do Departamento de Enfermagem Médico-Cirúrgico (MEM/EEAAC/UFF) Vice-coordenadora da Pós-Graduação em Enfermagem Gerontológica (EEAAC/UFF). Líder do NEPEG. Rio de Janeiro, RJ. Brasil. E-mail: rosifesa@gmail.com, ${ }^{3}$ Enfermeira. Doutora em Enfermagem. Professora Adjunta MEM/EEAAC/UFF. Coordenadora da Pós-Graduação em Enfermagem Gerontológica (EEAAC/ UFF). Vice-líder NEPEG. Rio de Janeiro, R. Brasil. E-mail: fatahelen@terra.com.br, ${ }^{4}$ Bolsista PIBIC. Acadêmica de Enfermagem do $8^{\circ}$ período do curso de graduação e licenciatura EEAAC/UFF. Rio de Janeiro, R. Brasil. E-mail: jany19rj@yahoo.com.br, ${ }^{5}$ Enfermeira. Residente em Enfermagem do Instituto Nacional do Câncer. Especializanda em Enfermagem Gerontológica (EEAAC/UFF). Bolsista voluntária do projeto. Rio de Janeiro, R. Brasil. E-mail: luise_almeida@yahoo.com.br 


\section{INTRODUÇÃO}

Ocorre uma predominância do paradigma biomédico na assistência hospitalar, com um desenho institucional determinado por especialidades médicas destinadas a tratamento de doenças, direcionadas, em geral, a um público adulto. ${ }^{1,2}$ Assim, o desenho atual das clínicas hospitalares dificulta distinguir as especificidades e particularidades esperadas no processo de envelhecimento humano, fato esse prioritário, já que atualmente presenciamos uma 'geriatrização' do cenário hospitalar brasileiro. ${ }^{3}$

Assim, corremos o risco de dispensá-los uma atitude carregada por mitos e estereótipos, desenvolvendo um cuidado generalizado tendo como base os parâmetros da fase adulta, impedindo o alavancar de uma área em formação e expansão: a enfermagem gerontológica, juntamente com toda área gerontogeriátrica. ${ }^{1-6}$ Portanto, torna-se indispensável entendermos o problema das síndromes geriátricas no ambiente hospitalar e suas implicações para o cuidado de enfermagem, delimitando como questão norteadora: Quais as relações entre os diagnósticos de enfermagem e as síndromes geriátricas?

As consequências fisiológicas e patológicas do envelhecimento, quando negligenciadas contribuem sobremaneira para 0 aparecimento das grandes síndromes geriátricas, ou seja, os " 5 is" da geriatria: Instabilidade Postural, Incontinência Urinária, Insuficiência Cerebral, latrogenia e Isolamento Social., ${ }^{7,8}$

Muitas vezes estas síndromes podem ser consideradas erroneamente como alterações normais do envelhecimento, aumentando consequentemente a cronicidade dos idosos, daí a necessidade de se investigar os diagnósticos de enfermagem associados a tal fenômeno, já que consideramos a sistematização da assistência de enfermagem uma meta ao processo individualizado, holístico, planejado, contínuo e avaliado, de modo a tornar o atendimento específico e integral ao cliente idoso. ${ }^{9,10}$ Para tanto, traçamos como objetivos: Identificar e Analisar os diagnósticos de enfermagem relacionados à presença das síndromes geriátricas em idosos hospitalizados.

Ao realizarmos uma busca no estado da arte por meio de descritores idoso e diagnóstico de enfermagem, encontramos cinco artigos versando sobre diagnósticos de enfermagem presentes em idosos hospitalizados dentre os 44 presentes no LILACS e os 208 presentes no MEDLINE, porém estes não analisavam sua relação com as síndromes geriátricas. Estas são analisadas de forma distinta ao discurso de ação da enfermagem e gerontologia.

Assim, ao traçarmos o perfil diagnóstico da clientela idosa internada em unidades clínicas e cirúrgicas e sua correlação com as síndromes geriátricas, pretendemos alcançar uma prática sistematizada de enfermagem, visando uma assistência adequada às necessidades reais $\mathrm{e}$ potenciais, contribuindo de forma efetiva para a recuperação e manutenção da saúde e esperando contribuir para a redução da incidência de complicações e o tempo de hospitalização e reinternações do cliente idoso.

\section{MÉTODO}

Trata-se de um estudo quantitativo prospectivo, para atender o objetivo de acompanhamento do evento em sua evolução. Para tanto, utilizamos como técnica de coleta de dados a entrevista semiestruturada e formulários. Os seguintes instrumentos de coleta de dados foram selecionados: a consulta de enfermagem gerontológica ${ }^{5}$; MEEM - Miniexame do Estado Mental; Lawton - Escala de Atividade de Instrumental Vida Diária; Katz - Escala de Atividade de Vida Diária e EDG - Escala de Depressão Geriátrica. ${ }^{8,11}$ Estes foram escolhidos tendo em vista a necessidade de uma avaliação dos problemas subjacentes à queixa principal, incluindo o funcional, o cognitivo, a emocional, a nutricional e o social, entendendo que estas interferem diretamente na saúde e no bem-estar, e principalmente na capacidade funcional do indivíduo idoso, ou seja, no grau de autonomia e independência.

Para seleção dos sujeitos, tivemos como critérios: ter 60 anos completos, ter acompanhante responsável e concordar em participar do estudo. Foram excluídos: sujeitos com menos de 60 anos, sem condições clínicas estáveis, sem acompanhantes responsáveis e que concordassem em participar da pesquisa. Para tanto, tivemos como período da coleta de dados janeiro de 2008 a março de 2009, garantindo uma coleta de dados longitudinal durante o tempo de internação destes idosos e o contato com a família. Portanto, os sujeitos selecionados foram 66 idosos, sendo 34 da clínica médica e 32 da clínica cirúrgica do Hospital Universitário Antônio Pedro (HUAP) da Universidade Federal Fluminense - UFF.

$\mathrm{Na}$ análise dos dados utilizamos a estatística descritiva simples com apresentação dos dados em tabelas. E para a formulação diagnóstica, utilizamos o raciocínio clínico proposto por Risner ${ }^{9}$ (análise, julgamento e síntese dos dados) adotando a taxonomia $N_{N N D A}{ }^{10}$, a fim de identificar os principais diagnósticos de enfermagem. Por fim, relacionamos os diagnósticos encontrados nestes sujeitos com síndromes geriátricas.

Quanto aos aspectos éticos desse projeto de pesquisa, cabe ressaltar que este estudo cumpre as especificações éticas e legais da resolução 196/96 do Ministério da Saúde e obteve parecer favorável no Comitê de Ética local sob o número de protocolo 135/ 
08 CAAE 0102.0.258.000-08. Declaramos que a pesquisa não envolveu risco direto ao sujeito, e como possível benefício coube-nos realizar um processo reflexivo à medida que investigamos o tema e nos comprometemos a orientá-lo a qualquer dúvida. Quanto à entrevista, essa foi realizada de forma conveniente ao sujeito e respeitando seus limites.

\section{RESULTADOS}

Identificamos 394 diagnósticos de enfermagem (DE) relacionados às cinco principais síndromes geriátricas: Isolamento Social, 129 (32,8\%); latrogenia, 113 (28,6\%); Instabilidade Postural, 81 (20,6\%); Insuficiência cerebral, 44 (11,1\%); e Incontinência urinária, 27 (6,9\%).

A síndrome Isolamento Social foi a mais presente, não diferenciando-se expressivamente nas duas clínicas: foram 69 DE na clínica médica e 60 DE na clínica cirúrgica. Já as latrogenias ocorreram com maior frequência na clínica médica (68 DE contra 48 DE da clínica cirúrgica), e isso talvez se relacione ao uso de complexos regimes medicamentosos, pois ali foram encontrados 21 idosos com iatrogenia (com mais de oito medicamentos prescritos) contra 9 idosos na clínica cirúrgica. Foram similares os resultados correlatos à Instabilidade Postural (em ambas as clínicas foram encontrados idosos com Risco para Quedas, 13 na clínica médica e 11 na clinica cirúrgica), como também os problemas relacionados à mobilidade. Em relação à Insuficiência Cerebral, foi encontrado o dobro de pacientes com memória prejudicada, ou seja, 6 na clínica cirúrgica e 3 na clínica médica; tal fato se relaciona aos transtornos neurocirúrgicos e aos fatores relacionados a reações pós-anestésicos. E por último tivemos a Incontinência Urinária também mais evidente na clínica cirúrgica, 16 contra 11 na clínica médica, tendo como fatores relacionados as cirurgias urológicas e a sondagem vesical de demora no pós-cirúrgico e suas complicações.

\section{Diagnósticos de enfermagem relacionados ao Isolamento Social}

Relacionados à síndrome Isolamento social, tivemos os diagnósticos, respectivamente, nas clínicas médica e cirúrgica: dentição prejudicada $24(35 \%)$ e 21 (35\%), um distúrbio nos padrões de desenvolvimento/erupção dentário ou na integridade estrutural dos dentes de um indivíduo; déficit sensorial: visão $12(17 \%)$ e 9 (15\%); déficit sensorial: audição $3(5 \%)$, nas duas clínicas; memória prejudicada $3(5 \%)$ e $6(10 \%)$; comunicação verbal prejudicada $3(9 \%)$ em ambas as clínicas; dor aguda 5 (7\%) nas duas clínicas, entendida como uma experiência sensorial e emocional desagradável que surge de lesão tissular real ou potencial ou descrita, em termos de tal lesão de início súbito ou lento, de intensidade leve a intensa, com término antecipado ou previsível e duração de menos de seis meses; estas, além de proporcionarem desconforto, diminuem as habilidades interativas do idoso; ansiedade $2(3 \%)$ e $3(5 \%)$, um vago e incômodo sentimento de desconfor to ou temor, acompanhado por resposta autonômica e sentimento de apreensão causado pela antecipação de perigo, que também contribuem para o afastamento social do idoso; tristeza crônica, identificada em 1 (3\%) idoso em cada clínica, está compreendida como o padrão cíclico, recorrente e potencialmente progressivo de tristeza disseminada, que é experimentada em resposta à perda contínua ao longo da trajetória de uma doença ou deficiência. Apenas na clínica médica foram encontrados 2 (3\%) idosos com disfunção sexual relacionada ao estado em que 0 indivíduo passa por mudança na função sexual, durante as fases de resposta sexual de desejo, excitação e/ou orgasmo, que é vista como insatisfatória, não compensadora e inadequada, que pode prejudicar sua interação com o mundo; quanto a padrões de sexualidade ineficazes, 1 $(1 \%)$ e $2(3 \%)$ apresentavam expressões de preocupação quanto à sua própria sexualidade; a baixa autoestima situacional, em 1 (3\%) idoso em cada clínica, entende-se como o desenvolvimento de percepção negativa sobre o seu próprio valor em resposta a uma situação atual; $1(1 \%)$ e $2(3 \%)$ apresentavam desesperança, estado subjetivo no qual um indivíduo não enxerga alternativas ou escolhas pessoais disponíveis ou enxerga alternativas limitadas, sendo incapaz de mobilizar energias a seu favor e também na interação social; 1 (3\%), somente na clínica cirúrgica, encontrava-se com risco para baixa autoestima situacional; 9 (13\%) e $3(5 \%)$ com atividade de recreação deficiente e $2(\%)$ apresentavam disposição para comunicação aumentada, na clínica médica. Na Tabela 1 apresentamos os principais diagnósticos de enfermagem relacionados à Síndrome Isolamento Social. 
Tabela 1. Principais diagnósticos de enfermagem encontrados nos idosos internados nas clínicas médica e cirúrgica (n-66) relacionados à síndrome geriátrica Isolamento Social. EEAAC/HUAP, Niterói-RJ, 2008-2009.

\begin{tabular}{|c|c|c|c|c|c|c|c|}
\hline \multirow{2}{*}{$\begin{array}{l}\text { Síndromes } \\
\text { Geriátricas }\end{array}$} & \multirow[t]{2}{*}{ Diagnósticos de Enfermagem } & \multicolumn{2}{|c|}{ Cínica Médica } & \multicolumn{2}{|c|}{ Clínica Cirúrgica } & \multicolumn{2}{|c|}{ Total } \\
\hline & & n (34) & $\%$ & n (32) & $\%$ & $\mathrm{n}(66)$ & $\%$ \\
\hline \multirow{15}{*}{$\begin{array}{l}\text { Is olamento } \\
\text { Social }\end{array}$} & Dentição Prejudicada & 24 & 35 & 21 & 35 & 45 & 35 \\
\hline & Déficit Sensorial: visão & 12 & 17 & 9 & 15 & 21 & 16 \\
\hline & Déficit Sensorial: audição & 3 & 5 & 3 & 5 & 6 & 5 \\
\hline & Memória Prejudicada & 3 & 5 & 6 & 10 & 9 & 7 \\
\hline & $\begin{array}{l}\text { Comunicação Verbal } \\
\text { Prejudicada }\end{array}$ & 3 & 5 & 3 & 5 & 6 & 5 \\
\hline & Dor Aguda & 5 & 7 & 5 & 8 & 10 & 8 \\
\hline & Ansiedade & 2 & 3 & 3 & 5 & 6 & 5 \\
\hline & Tristeza Crônica & 1 & 1 & 1 & 2 & 2 & 1 \\
\hline & Disfunção Sexual & 2 & 3 & - & - & 2 & 1 \\
\hline & $\begin{array}{l}\text { Padrões de Sexualidade } \\
\text { Ineficazes }\end{array}$ & 1 & 1 & 2 & 3 & 3 & 3 \\
\hline & Baixa Autoestima Situacional & 1 & 1 & 1 & 2 & 2 & 1 \\
\hline & Desesperança & 1 & 1 & 2 & 3 & 3 & 3 \\
\hline & $\begin{array}{l}\text { Risco para Baixa Autoestima } \\
\text { Situacional }\end{array}$ & - & - & 1 & 2 & 1 & 0 \\
\hline & $\begin{array}{l}\text { Atividade de Recreação } \\
\text { Deficiente }\end{array}$ & 9 & 13 & 3 & 5 & 12 & 9 \\
\hline & $\begin{array}{l}\text { Disposição para Comunicação } \\
\text { Aumentada }\end{array}$ & 2 & 3 & - & - & 2 & 1 \\
\hline \multicolumn{2}{|r|}{ Total } & 69 & 100 & 60 & 100 & 129 & 100 \\
\hline
\end{tabular}

Estes diagnósticos fazem parte tanto desta síndrome como da de insuficiência cerebral, pois a perdas das habilidades cognitivas podem contribuir tanto com dificuldades mnemônicas e a determinados estímulos, como também auxilia no afastamento e perda do interesse na realização e participação de atividades culturais e sociais.

\section{Diagnósticos de enfermagem relacionados à latrogenia}

Os diagnósticos relacionados com a latrogenia estão apresentados na Tabela 2; respectivamente, temos nas clínicas médica e cirúrgica: 7 (11\%) idosos com risco de infecção, entendido como o risco aumentado de ser invadido por organismos patogênicos, presente em ambas as clínicas; $2(3 \%)$ e $6(13 \%)$ com nutrição desequilibrada: menos que as necessidades corporais; 2 (3\%) e 1 (2\%) com hipertermia; 1 ( $1 \%$ e $2 \%$ ) com hipotermia; $2(3 \%$ e $4 \%$ ) com risco de glicemia instável; $3(5 \%$ e $6 \%)$ com volume de líquido deficiente; 9 (14\% e $19 \%$ ) com risco de integridade da pele prejudicada, nas duas clínicas; $6(9 \%)$ e $1(2 \%)$ apresentavam integridade da pele prejudicada; $7(11 \%)$ e 2 (4\%) com manutenção ineficaz da saúde: preventivo; $9(14 \%)$ e $6(13 \%)$ com manutenção ineficaz da saúde: gripe; 5 (8\%) e 1 (2\%) encontravam-se com o diagnóstico de manutenção ineficaz da saúde: próstata; 1 (1\% e $2 \%$ ) idoso com diarreia; 3 (5\% e 6\%) com risco de constipação em ambas; $8(12 \%)$ e $5(10 \%)$ com constipação. 
Tabela 2. Principais diagnósticos de enfermagem encontrados nos idosos internados nas clínicas médica e cirúrgica (n-66) relacionados à síndrome geriátrica latrogenia. EEAAC/HUAP, Niterói-RJ, 2008-2009.

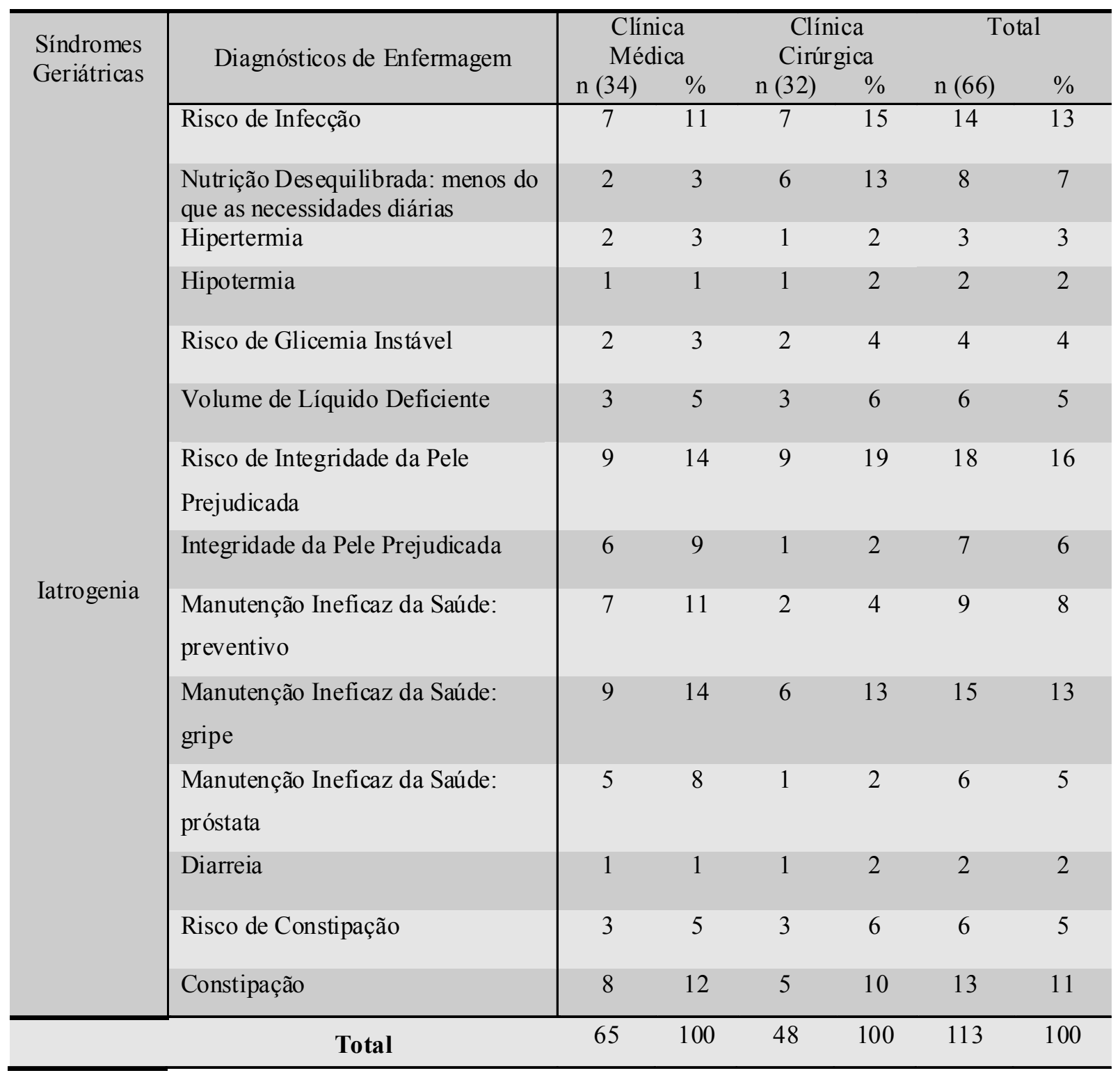

Os diagnósticos relacionados contribuem para 0 aumento da gravidade e/ou ocorrência desta, principalmente por estarem associados às doenças crônicas geralmente existentes, e agravam o quadro clínico subjacente à hospitalização.

Diagnósticos de enfermagem relacionados à Instabilidade Postural

A instabilidade postural é um dos principais problemas que afeta a vida dos idosos e que pode desencadear outros problemas, como dificuldade na deambulação, risco de queda, imobilidade, baixa autoestima, desenvolvimento de medo e insegurança. $^{8}$

Os diagnósticos de enfermagem relacionados à síndrome geriátrica Instabilidade Postural serão apresentados respectivamente na clínica médica e cirúrgica: o Risco para quedas observou-se em 13 (38\%) e 11 (34\%), entendido como uma suscetibilidade aumentada para quedas que podem causar danos físicos; deambulação prejudicada, $7(20 \%)$ e $4(12 \%)$, a limitação à movimentação independente, a pé, pelo ambiente; mobilidade física prejudicada, 4 $(12 \%)$ e $6(19 \%)$, a limitação no movimento físico independente e voluntário do corpo ou de uma ou mais extremidades; insônia, 6 (18\%) e $5(15 \%)$, o distúrbio na quantidade e na qualidade do sono que prejudica o funcionamento normal de uma pessoa; débito cardíaco diminuído, $3(9 \%)$ e 1 (3\%), a quantidade insuficiente de sangue bombeado pelo coração para atender às demandas metabólicas corporais; intolerância à atividade, $2(6 \%)$ e 1 ( $3 \%$ ), energia fisiológica ou psicológica insuficiente para suportar ou completar as atividades diárias requeridas ou desejadas; 
déficit no autocuidado: alimentação, banhol higiene, $2(6 \%)$ e 6 (19\%); estilo de vida sedentário, 2 (6\%) e 1 (3\%); perfusão tissular ineficaz não foi encontrada na clínica médica e observou-se em 1 (3\%) na cirúrgica; e padrão respiratório ineficaz, $4(12 \%)$ e $2(6 \%)$. Na Tabela 3 apresentamos a relação entre os Diagnósticos de Enfermagem, segundo NANDA, agrupados de acordo com a síndrome geriátrica Instabilidade postural.

Tabela 3. Principais diagnósticos de enfermagem encontrados nos idosos internados nas clínicas médica e cirúrgica (n-66) relacionados à síndrome geriátrica Instabilidade Postural. EEAAC/HUAP, Niterói-RJ, 2008-2009.

\begin{tabular}{|c|c|c|c|c|c|c|c|}
\hline \multirow{2}{*}{$\begin{array}{l}\text { Sindrome } \\
\text { Geriátrica }\end{array}$} & \multirow{2}{*}{ Diagnósticos de Enfermagem } & \multicolumn{2}{|c|}{$\begin{array}{l}\text { Clínica } \\
\text { Médica }\end{array}$} & \multicolumn{2}{|c|}{$\begin{array}{c}\text { Clínica } \\
\text { Cirúrgica }\end{array}$} & \multicolumn{2}{|c|}{ Total } \\
\hline & & $\mathrm{n}(34)$ & $\%$ & $\mathrm{n}(32)$ & $\%$ & $\mathrm{n}(66)$ & $\%$ \\
\hline \multirow{10}{*}{$\begin{array}{l}\text { Instabilidade } \\
\text { Postural }\end{array}$} & Risco para Quedas & 13 & 30 & 11 & 29 & 24 & 29 \\
\hline & Deambulação Prejudicada & 7 & 16 & 4 & 11 & 11 & 14 \\
\hline & Mobilidade física Prejudicada & 4 & 9 & 6 & 15 & 10 & 12 \\
\hline & Insônia & 6 & 14 & 5 & 13 & 11 & 14 \\
\hline & Débito Cardíaco Diminuído & 3 & 7 & 1 & 3 & 4 & 5 \\
\hline & Intolerância à Atividade & 2 & 5 & 1 & 3 & 3 & 4 \\
\hline & $\begin{array}{l}\text { Déficit no Auto-Cuidado: } \\
\text { alimentação, banho/higiene }\end{array}$ & 2 & 5 & 6 & 15 & 8 & 10 \\
\hline & Estilo de Vida Sedentário & 2 & 5 & 1 & 3 & 3 & 4 \\
\hline & Perfusão Tissular Ineficaz & - & - & 1 & 3 & 1 & 1 \\
\hline & Padrão Respiratório Ineficaz & 4 & 9 & 2 & 5 & 6 & 7 \\
\hline & Total & 43 & 100 & 38 & 100 & 81 & 100 \\
\hline
\end{tabular}

Em relação à Instabilidade Postural, pôde ser observado que esta se mostra em igual número em ambas clínicas, elucidando a necessidade de se observar os diagnósticos presentes nos idosos que a caracterizam e que não há diferenciação no aparecimento destes com relação à finalidade da internação, queixa principal ou fatores agravantes de cada clínica.

Diagnósticos de enfermagem relacionados à Instabilidade Cerebral

Relacionados à Insuficiência Cerebral, foram encontrados nas clínicas médica e cirúrgica, respectivamente os seguintes diagnósticos: $12(35 \%)$ e $9(28 \%)$ idosos com déficit sensorial: visão; $3(9 \%)$ em ambas as clínicas com déficit sensorial: audição. Déficit sensorial é a mudança na quantidade e no padrão dos estímulos que estão sendo recebidos, acompanhada por respostas diminuída, exagerada, distorcida ou prejudicada a tais estímulos. $3(9 \%)$ e
$6(19 \%)$ dos idosos tinham memória prejudicada, que é a incapacidade de lembrar ou recordar partes de informação ou habilidades comportamentais; em ambas as clínicas $3(9 \%)$ apresentavam comunicação verbal prejudicada: habilidade diminuída, retardada ou ausente para receber, processar, transmitir e usar um sistema de símbolos. E somente na clínica cirúrgica 1 (3\%) idoso apresentava confusão aguda: início abrupto dos distúrbios reversíveis de consciência, atenção, cognição e percepção que ocorrem durante um breve período de tempo; e outro (3\%), confusão crônica: uma deterioração irreversível, prolongada e/ou progressiva do intelecto e da personalidade, caracterizada por capacidade diminuída para a interpretação dos estímulos ambientais e para processos de pensamento intelectual, e manifestada por distúrbios da memória, da orientação e do comportamento. Na Tabela 4 apontamos os principais diagnósticos de enfermagem relacionados à Síndrome Insuficiência Cerebral. 
Tabela 4. Principais diagnósticos de enfermagem encontrados nos idosos internados nas clínicas médica e cirúrgica (n-66) relacionados à síndrome geriátrica Insuficiência Cerebral. EEAAC/HUAP, Niterói-RJ, 2008-2009.

\begin{tabular}{|c|c|c|c|c|c|c|c|}
\hline \multirow{2}{*}{$\begin{array}{l}\text { Síndromes } \\
\text { Geriátricas }\end{array}$} & \multirow[t]{2}{*}{ Diagnósticos de Enfermagem } & \multicolumn{2}{|c|}{$\begin{array}{l}\text { Clínica } \\
\text { Médica }\end{array}$} & \multicolumn{2}{|c|}{$\begin{array}{l}\text { Clínica } \\
\text { Cirúrgica }\end{array}$} & \multicolumn{2}{|c|}{ Total } \\
\hline & & n (34) & $\%$ & $\mathrm{n}(32)$ & $\%$ & $n(66)$ & $\%$ \\
\hline \multirow{6}{*}{$\begin{array}{c}\text { Insuficiência } \\
\text { Cerebral }\end{array}$} & Déficit Sensorial: visão & 12 & 58 & 9 & 40 & 21 & 48 \\
\hline & Déficit Sensorial: audição & 3 & 14 & 3 & 13 & 6 & 14 \\
\hline & Memória Prejudicada & 3 & 14 & 6 & 26 & 9 & 20 \\
\hline & $\begin{array}{l}\text { Comunicação Verbal } \\
\text { Prejudicada }\end{array}$ & 3 & 14 & 3 & 13 & 6 & 14 \\
\hline & Confusão Aguda & - & - & 1 & 4 & 1 & 2 \\
\hline & Confusão Crônica & - & - & 1 & 4 & 1 & 2 \\
\hline & Total & 21 & 100 & 23 & 100 & 44 & 100 \\
\hline
\end{tabular}

Como pôde ser visto no resultado da aplicação do MEEM, os resultados foram satisfatórios em sua maioria, evidenciando um número mínimo de idosos com comprometimento de cognição, julgamento e memória. A avaliação cognitiva auxilia na identificação das principais alterações na saúde mental das pessoas idosas; devem ser identificados sinais e sintomas precocemente, pois durante a hospitalização podem indicar uma complicação no quadro clínico. 0 desempenho físico e social do idoso depende da integridade de suas funções cognitivas. A perda da memória recente e a habilidade de cálculo são indicadores sensíveis de redução dessas funções. ${ }^{9}$

\section{Diagnósticos de enfermagem relacionados à Incontinência Urinária}

Relacionados à Incontinência Urinária foram encontrados, respectivamente na clínica médica e cirúrgica, 10 (29\%) e 14 (43\%) idosos com diagnóstico de eliminação urinária prejudicada, que é um distúrbio na eliminação de urina; $1(3 \%)$ e $2(6 \%)$ com incontinência urinária funcional: incapacidade da pessoa que é usualmente continente e pode alcançar o banheiro a tempo de evitar perda de urina. 0 envelhecimento por si só não é causa de incontinência urinária, mas induz algumas mudanças funcionais e estruturais no sistema urinário, que podem predispor à incontinência. ${ }^{10}$ Existem características definidoras dos diagnósticos encontrados nos idosos que reforçam a relação existente deste com esta síndrome, tais como a perda de urina antes de alcançar o banheiro relacionado à estrutura de suporte pélvicas enfraquecidas, pois o que ocorre em ambos os sexos é um declínio da habilidade para retardar a micção. ${ }^{10}$ Antes mesmo de considerar a incontinência inevitável é preciso haver investigações de suas causas, para que este problema não seja considerado um processo comum do envelhecimento. Pacientes idosos hospitalizados, não prontamente atendidos pelo corpo de enfermagem ou por cuidador durante o desejo de urinar, podem tornar-se, com o tempo, funcionalmente incontinentes. ${ }^{10}$ Apresentamos na Tabela 5 os principais diagnósticos de enfermagem encontrados nos idosos relacionados à Síndrome Incontinência Urinária.

Tabela 5. Principais diagnósticos de enfermagem encontrados nos idosos internados nas clínicas médica e cirúrgica (n-66) relacionados à síndrome geriátrica Incontinência Urinária. EEAAC/HUAP, Niterói-RJ, 2008-2009.

\begin{tabular}{|c|c|c|c|c|c|c|c|}
\hline \multirow{2}{*}{$\begin{array}{l}\text { Síndrome } \\
\text { Geriátrica }\end{array}$} & \multirow{2}{*}{$\begin{array}{l}\text { Diagnósticos de } \\
\text { Enfermagem }\end{array}$} & \multicolumn{2}{|c|}{ Clínica Médica } & \multicolumn{2}{|c|}{$\begin{array}{l}\text { Clínica } \\
\text { Cirúrgica }\end{array}$} & \multicolumn{2}{|c|}{$\begin{array}{l}\text { Clínica } \\
\text { Cirúrgica }\end{array}$} \\
\hline & & $\mathrm{n}(34)$ & $\%$ & n (32) & $\%$ & $\mathrm{n}(66)$ & $\%$ \\
\hline \multirow{2}{*}{$\begin{array}{l}\text { Incontinência } \\
\text { Urinária }\end{array}$} & $\begin{array}{c}\text { Eliminação Urinária } \\
\text { Prejudicada }\end{array}$ & 10 & 91 & 14 & 88 & 24 & 89 \\
\hline & $\begin{array}{c}\text { Incontinência Urinária } \\
\text { Funcional }\end{array}$ & 1 & 9 & 2 & 12 & 3 & 11 \\
\hline \multicolumn{2}{|r|}{ Total } & 11 & 100 & 16 & 100 & 27 & 100 \\
\hline
\end{tabular}


A incontinência urinária do ponto de vista psicológico é também grave fator de descompensação em idosos, nos quais a sensação de rejeição, infelizmente, corresponde, muitas vezes, à realidade, devido ao inconveniente social acarretado pela impossibilidade de se manter limpo, prejudicando o autocuidado e autoestima. ${ }^{11}$

\section{DISCUSSÃO}

Com os diagnósticos estabelecidos, foi possível observar a presença das síndromes geriátricas nos idosos abordados; a denominação 'idoso frágil' será utilizada para descrever aqueles idosos que se encontram em risco mais elevado para resultados de saúde adversos ou síndromes geriátricas. ${ }^{8}$ Esta pode estar associada a imunidade reduzida dos idosos, contribuindo para o aparecimento de uma lesão ou doença, ou seu agravamento.

Independentemente de ser expressa de forma aguda ou crônica, a doença apresentada pelo idoso é multifatorial e dificilmente resulta de uma única causa, daí a complexidade do cuidado de enfermagem dispensado a essa clientela e a necessidade de uma equipe especializada para esse atendimento, pois, comumente, os sintomas surgem de forma aguda e podem-se ter sistemas corporais afetados antes mesmo do surgimento dos sintomas.

Portanto, se considerarmos a presença dos 394 diagnósticos de enfermagem encontrados nos 66 idosos investigados, calculamos que, em média, duas síndromes ocorrem por idoso, que sofre o risco de fragilização durante o processo de hospitalização, com aumento da chance de complicações, do custo e das reinternações. Isso merece uma atenção integral e holística, com adequação de um plano de cuidados contínuos, a curto, médio e longo prazo, intencionalmente preparado para a promoção prevenção e manutenção da capacidade funcional.

Os dados descritos revelam que os idosos estão sendo internados com os diagnósticos que caracterizam a presença de síndromes geriátricas, e que muitas vezes saem do hospital apresentado os mesmos diagnósticos que possuíam quando entraram nele, ou ainda mais, adquiridos durante o período de internação. Isso reforça a afirmação já feita e que será defendida no decorrer do artigo de que as síndromes favorecem o aparecimento da fragilidade nos idosos levando a uma série de debilitações que acarretem a dependência. E reforça a importância do cuidado direcionado às necessidades de cada idoso.

Os diagnósticos relacionados ao Isolamento Social podem desencadear baixa autoestima, desmotivação, diminuição do autocuidado, causando tristeza crônica e dependência desse idoso e, com isso, levar ao surgimento de doenças crônicas e fatores incapacitantes. ${ }^{12} \mathrm{~A}$ autoestima é um fator de suma importância que pode ajudar a desenvolver ou a manter a dependência ou a independência da pessoa idosa e influencia no desenvolvimento de doenças crônicas, perda da capacidade de mobilidade, hospitalização e avançar da idade, que contribuem para um maior risco de desenvolver a baixa autoestima. Por isso as ações de enfermagem devem se centrar em estimulá-los a realizar as atividades de autocuidado, para ganhar mais independência e a participar das decisões que afetam direta ou indiretamente sua qualidade de vida e bemestar.

A iatrogenia adquire importância tanto pela incidência como por a intensidade de suas manifestaç̃̃es serem mais acentuadas nos idosos. ${ }^{11} 0$ idoso ao ser hospitalizado pode estar sujeito a iatrogenias, sendo o principal tipo a reação adversa a medicamentos, definida como a resposta nociva e não intencional ao uso de um ou mais medicamentos, que ocorre em associações de doses normalmente empregadas para profilaxia, diagnóstico e tratamento.

0 fato de na clínica médica a síndrome iatrogênica ter sido encontrada em um maior número de idosos, como vimos, faz-nos observar que, nela, o idoso permanece internado por mais tempo e por isso é exposto aos medicamentos por mais tempo. Na clínica cirúrgica, geralmente os pacientes são internados, realizam a cirurgia, se recuperam em um período brevemente estabelecido, se não houver nenhuma intercorrência, e logo recebem alta hospitalar. Soma-se a isso o fato de os idosos estarem na clínica médica para compensar as doenças crônicas, geralmente mais de uma, e por isso possuírem a necessidade de maior número de medicamentos associados.

Esta reação pode causar o prolongamento da internação e com isso aumentar ainda mais a suscetibilidade dos idosos ao risco de infecção por se elevar a disposição ambiental a patógenos e procedimentos invasivos. As pessoas idosas correspondem ao grupo etário que mais utilizam medicamentos, principalmente aqueles de venda livre. ${ }^{10}$ Os medicamentos melhoram a saúde e o bem-estar da pessoa idosa; no entanto, os problemas ocorrem com frequência por causa das interações medicamentosas; múltiplos efeitos dos medicamentos; uso de múltiplos medicamentos (polifarmácia); e falta de adesão rigorosa ao regime prescrito.

As combinações dos medicamentos prescritos e alguns de venda livre complicam ainda mais o problema. ${ }^{6}$ Reações adversas podem ser definidas como fator previsivel de morbidade e mortalidade; leva-se em conta não só sua elevada prevalência na população idosa, mas também os custos gerados e sua importância epidemiológica como possível causa de internação. É notória a importância dos profissionais de saúde em identificálas e tratá-las de forma adequada, essa entendida dentro de uma equipe multi e interprofissional. ${ }^{10}$

As grandes propensões da pessoa idosa à instabilidade postural e à alteração da marcha aumentam o risco de quedas. As quedas representam um sério problema para as pessoas idosas e estão associadas aos elevados índices de morbimortalidade, redução da capacidade funcional e 
institucionalização precoce, ${ }^{9}$ visto que as quedas têm como complicações o surgimento do medo e o desencadeamento de traumas e fraturas, diminuição das atividades, podendo acarretar dependência e morte. Isso mostra a necessidade de a equipe de enfermagem atuar no estímulo à atividade física e nutrição adequada, realizar uma avaliação de riscos domésticos, a revisão periódica dos medicamentos utilizados e correção dos déficits sensoriais. ${ }^{10}$

Esses dados confirmam o resultado obtido na pesquisa e elucida a importância de o profissional de saúde observar e prevenir a ocorrência de quedas, e avaliar a marcha da pessoa idosa a fim detectar alguma alteração comprometedora. Nos casos em que a pessoa está com a mobilidade extremamente debilitada, como no caso de pessoas acamadas por longos períodos, são necessárias medidas que estimulem os idosos a permanecer o menor tempo possível de repouso no leito. Quando isto for inevitável, o paciente deve realizar os exercícios de amplitude de movimentos ativos e de fortalecimento com os membros sadios, e a enfermeira deve realizar os exercícios de amplitude de movimento passivos nos membros afetados, ${ }^{6}$ até que 0 idoso possa transferir-se da cama para a cadeira e deambular com o auxílio de outra pessoa ou de bengalas, andadores, muletas ou cadeira de rodas.

Entendemos que o processo de envelhecimento é acompanhado pela perda gradual das funções cognitivas aliada a processos neurológicos que se modificam com a idade. São considerados normais a "lentificação e a dificuldade de armazenamento de novas informações", portanto, são exemplos a dificuldade de recordar números de telefone, nome de pessoas e objetos guardados; porém, a perda de memória e a perda da capacidade de executar as funções executivas progressivamente associadas à agnosia, à afasia e à apraxia são consideradas alterações não esperadas no processo de envelhecimento e merecem atenção da enfermagem, pois a omissão desses sinais e a não intervenção nos diagnósticos relacionados a essa síndrome podem ocasionar uma piora no quadro, ainda mais associado à hospitalização.

Esse declínio varia de acordo com fatores intelectuais, educacionais e com o estado de saúde de cada idoso. Portanto, é necessário que os profissionais de saúde estejam atentos ao surgimento desses sinais para que, precocemente, seja feita a intervenção e se possa evitar sofrimento aos idosos, bem como a seus familiares. Para tanto, devem ser feitas, além do acompanhamento no hospital quando o paciente estiver internado, orientações aos familiares, numa perspectiva integral e gerontológica.

A incontinência urinária foi observada em maior número na clínica cirúrgica. Pode-se associar isso ao fato de muitos dos idosos estarem lá justamente para corrigir alguma disfunção do trato urinário e por se encontrarem menos ativos logo após a cirurgia.
Essa síndrome deve ser avaliada, pois cerca de $30 \%$ das pessoas idosas não institucionalizadas costumam apresentá-la e nem sempre a referem na avaliação clínica, ou por vergonha ou por acharem que seja normal no processo de envelhecimento ${ }^{9}$. Visto isso, evidencia-se que o enfermeiro é fundamental na prevenção e reabilitação da incontinência, por meio do planejamento, execução e educação dos técnicos de enfermagem e dos cuidadores através de medidas não farmacológicas. Dentre estas incluem facilitação do acesso ao banheiro, adaptação da altura dos vasos, instalação de barras de apoio, iluminação adequada e uso de urinóis ou cadeira sanitária à beira do leito. Além disso, outras medidas para evitar episódios de incontinência podem ser implementadas, tais como o uso de roupas que possam ser abertas com facilidade, micção estimulada e em horário regulado e cateterismo intermitente. ${ }^{6}$

\section{CONCLUSÃO}

Pode ser observada, durante a hospitalização, a necessidade de um planejamento da assistência de enfermagem gerontológica, pois, com a identificação dos 394 diagnósticos de enfermagem, podemos inferir que estes contribuem para o surgimento e o agravamento do quadro de comorbidades, resultando em aumento de hospitalizações e complicações, tornando complexos os casos, requerendo, assim, da enfermagem atenção especializada gerontogeriátrica.

Assim, o idoso deve ser atendido em suas necessidades biopsicossociais, com base em uma avaliação multidimensional gerontogeriátrica, identificando-se as necessidades físicas, funcionais, mentais e sociais e relacionando-as a possíveis síndromes geriátricas. Tal fato, pode auxiliar a enfermeira a comunicar de forma clara e objetiva os cuidados de enfermagem necessários aos membros da equipe profissional, já que as síndromes geriátricas fazem parte de um corpo de conhecimento específico da gerontogeriatria, contribuindo ademais na construção de uma linguagem própria na enfermagem gerontogeriátrica.

Demonstramos também que o cuidado de enfermagem para essa população propõe desafios complexos com uma gama de necessidades na saúde física, funcional, cognitiva e psicossocial. Assim, os idosos apresentaram necessidades médicas e sociais diferenciadas e tenderam à utilização de forma mais intensiva dos serviços e equipamentos de saúde; por isso, uma atenção integral deve ser garantida para diminuir tais índices, visto que o isolamento social esteve mais evidente, apesar de suas relações serem prioritariamente de ordem física e mental.

Neste sentido, é fundamental que os profissionais da área da saúde, em especial a equipe de enfermagem, tenha conhecimento das particularidades inerentes aos idosos e ao processo de envelhecimento para que os sinais e sintomas 
característicos do surgimento de uma síndrome geriátrica sejam previamente diagnosticados, principalmente durante a hospitalização, a fim de evitar a progressão do quadro, proporcionando planos terapêuticos que garantam sua execução em curto, médio e longo prazo, considerando um continuum de cuidado, desde a promoção, prevenção, tratamento e reabilitação adequada das suas funções.

\section{REFERÊNCIAS}

1. Wacker RR, Roberto KA. Heath care and wellness. In: Wacker RR, Roberto KA. Community resuources for older adults: programs and services in an era of chage. $3^{\mathrm{a}}$ ed. Califórnia (USA): Sage Publications; 2008.

2. Berger L. Cuidados de enfermagem em gerontologia. In: MaillouxPoirier M. Pessoas idosas: uma abordagem global. Lisboa(PT): Lusodidacta; 1995. p. 11-19.

3. Estrella K, Oliveira CEF, Sant'Anna AA, Caldas CP. Detecção do risco para internação hospitalar em população idosa: um estudo a partir da porta de entrada no sistema de saúde suplementar. Cad Saude Publica [on-line]. 2009 mar; [citado 2010 sep 29]; 25(3): [aprox.6 telas]. Disponível em http://www.scielo.br.

4. Touhy TA, Jett KF. Ebersole and Hess' Gerontological nursing healthy aging. $3^{\text {a }}$ ed. St. Louis(USA): Mosby Elsevier; 2010.

5. Eliopoulos C. Gerontological nursing. $7^{\text {a }}$ ed. Philadelphia(USA): Wolters Kluwer Health/ Lippincott Williams \& Wilkins; 2010.

6. Prochet TC, Silva MJP. Situações de desconforto vivenciadas pelo idoso hospitalizado com a invasão do espaço pessoal e territorial. Esc Anna Nery. 2008 jun; 12(2): 310-15.

7. Papaléo Neto M., Carvalho Filho ET. Geriatria: fundamentos, clínica e terapêutica. $2^{\mathrm{a}}$ ed. São Paulo (SP): Atheneu; 2006.

8. Ministério da Saúde (BR). Secretaria de Atenção a Saúde. Departamento de Atenção Básica. Caderno de Atenção Básica. Envelhecimento e saúde da pessoa idosa. Brasília (DF); 2006.

9. Risner P.B. Diagnosis: analysis and synthesis of data. In: Christesen P.J, Kenney JW Nursing process application of conceptual models. St. Louis(USA): Mosby; 1995.

10. NANDA-Diagnósticos de enfermagem da: definições e classificação: 2009-2010. Tradução de Cristina Correa. Porto Alegre (RS): Artmed; 2009.

11. Santana RF, Santos I. 0 cuidar do idoso dependente no contexto do Sistema Único de Saúde In: Santos I. Enfermagem e campos de prática em saúde coletiva.. Rio de Janeiro (RJ): Atheneu; 2008. v. 4, p. 345-51.
12. Piccoli M, Galvão C M. Enfermagem perioperatória: identificação do diagnóstico de enfermagem risco para infecção fundamentada no modelo conceitual de Levine. Rev Latino-am Enfermagem. 2001 jul; 9 (4): 37-43.

13. Barbosa MT. Como avaliar quedas em idosos? Rev. Assoc. Med Bras. [on line]. 2001 jun; [citado 2010 set 29]; 47(2): [aprox. 2 telas]. Disponível em: http://www.scielo.br.

14. Brucki SMD, Nitrini R, Caramelli P, Bertolucci PHF, Okamoto IH. Sugestões para o uso do mini-exame do estado mental no Brasil. Arq Neuro-Psiquiatr. [on-line] 2003 set [citado 2010 set 29]; 61(3B): [aprox.5 telas]. Disponível em http://www.scielo.br.

15. Veras R.P, et al. Avaliação dos gastos com o cuidado do idoso com demência. Rev. Psiquiatr. Clín. [on-line] 2007 [citado 2010 set 29]; 34(1): [aprox.5 telas]. Disponível em http://www.scielo.br.

16. Marin MJS, et al. Diagnósticos de enfermagem de idosas carentes de um programa de saúde da família (PSF). Esc Anna Nery. 2008 jun; 12(2): 278-84.

17. Figueiredo MLF, Luz MHBA, Brito CM, Souza SNS, Silva DRS. Diagnósticos de enfermagem do idoso acamado no domicílio. Rev Bras Enferm. 2008 ago; 61(4): 464-69.

18. Luciana MS, Duarte AYY. Diagnósticos e intervenções de enfermagem em idosos hospitalizados. Acta Paul Enferm. 2007; 20 (4): 495-98.

\section{NOTA}

a Recorte ampliado do projeto de pesquisa financiado pelo CNPq e FAPERJ "Atenção Integral à Saúde do Idoso internado em unidades clínicas e cirúrgicas: proposta de um modelo através dos princípios de enfermagem gerontológica.
Recebido em 05/05/2010 Reapresentado em 04/08/2010 Aprovado em 05/09/2010 